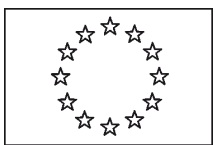

COMISIÓN DE LAS COMUNIDADES EUROPEAS

\author{
Bruselas, 11.9.2007 COM (2007) 512 final \\ COMUNICACIÓN DE LA COMISIÓN \\ AL CONSEJO, AL PARLAMENTO EUROPEO, \\ AL COMITÉ ECONÓMICO Y SOCIAL Y \\ AL COMITÉ DE LAS REGIONES
}

\title{
TERCER INFORME ANUAL SOBRE INMIGRACIÓN E INTEGRACIÓN
}

\section{INTRODUCCIÓN}

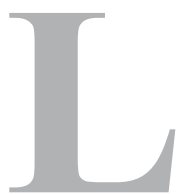

Los informes anuales ${ }^{1}$ sobre inmigración e integración analizan las medidas adoptadas relativas a la admisión e integración de nacionales de terceros países en los Estados miembros y en el conjunto de la UE, describen los avances y ayudan a evaluar y consolidar las medidas de integración.

Durante el pasado año, el debate sobre la integración se intensificó tanto en la UE como en los distintos países. Un número creciente de Estados miembros aplican nuevas políticas de integración y ajustan sus estrategias basándose en la experiencia acumulada.

Este tercer informe anual recoge lo acaecido hasta junio de $2007^{2}$; su anexo («Informe resumido sobre las políticas de integración en la UE-27»), elaborado en cooperación con los enlaces nacionales de integración ${ }^{3}$, cubre el año 2005 y el primer semestre de 2006.

\section{POBLACIÓN INMIGRANTE EN LA UE}

En enero de 2006, los nacionales de terceros países residentes en la UE suponían alrededor de 18,5 millones, es decir, el 3,8\% de una población total

1 El primer informe COM(2004) 508 se publicó en julio de 2004 y el segundo SEC (2006) 892, en junio de 2006.

2 El informe tiene en cuenta las conclusiones del Consejo de junio de 2007; doc. 10267/07 del Consejo.

3 La Comisión creó la red de enlaces nacionales para dar seguimiento a las conclusiones del Consejo JAI de octubre de 2002. 
de casi 493 millones $^{4}$. La inmigración sigue siendo el principal factor de crecimiento demográfico en la UE y en la mayor parte de los Estados miembros se registra una inmigración neta positiva ${ }^{5}$, que se situó entre 500.000 y un millón de personas anuales durante la mayor parte de los años noventa y aumentó a niveles de entre 1,5 y 2 millones desde 2002.

La tipología de la inmigración difiere mucho según el Estado miembro. Mientras que la reagrupación familiar es considerable en países como Austria, Francia o Suecia, otros Estados miembros, como Irlanda, España, Portugal y el Reino Unido, registraron un alto porcentaje de inmigración laboral ${ }^{6}$. En España se procedió a importantes regularizaciones mientras que Francia, Alemania y los Países Bajos optaron por regularizaciones limitadas para grupos específicos de inmigrantes.

Los grupos más numerosos de nacionales de terceros países en la UE proceden de Turquía (2,3 millones), Marruecos (1,7 millones), Albania ( 0,8 millones) y Argelia (0,6 millones). Sin embargo, el número de ciudadanos de Estados miembros como Francia, Suecia, los Países Bajos y el Reino Unido nacidos en el extranjero es mayor que el número de nacionales de terceros países, ya que muchos inmigrantes adquirieron la ciudadanía del país de acogida.

\section{DESARROLLO DE UNA POLÍTICA COMÚN DE INMIGRA- CIÓN Y MARCO DE LA UE PARA LA INTEGRACIÓN DE NA- CIONALES DE TERCEROS PAÍSES}

La integración de nacionales de terceros países es un proceso de adaptación mutua entre las sociedades de acogida y los inmigrantes y un factor esencial para obtener plenos beneficios de la inmigración. Tal como se subrayó en la Comunicación «El planteamiento global sobre la migración un año después: Hacia una política global europea en materia de migración» ${ }^{7}$, debe reforzarse continuamente el nexo entre políticas de inmigración legal y estrategias de integración.

La consolidación del marco jurídico relativo a las condiciones de entrada y estancia de nacionales de terceros países es esencial para desarrollar un planteamiento coherente de la UE con respecto a la integración. Ya existen instrumentos legislativos relativos a reunificación familiar, residentes de larga duración y normas relativas a los nacionales de terceros países o apátridas que precisan protección internacional ${ }^{8}$ que reconocen derechos como (depen-

4 Fuente: Eurostat. Aunque Rumanía y Bulgaria ingresaron en la UE en 2007, a efectos de esta estimación sus ciudadanos son considerados nacionales de la UE.

5 Excepto Estonia, Letonia, Lituania, Polonia y los Países Bajos. Eurostat, Resumen estadístico, Población y condiciones sociales, 1/2006.

6 OCDE, International Migration Outlook, Informe anual 2006.

$7 \operatorname{COM}(2006) 735$.

8 Directiva 2003/86 del Consejo sobre el derecho a la reagrupación familiar, Directiva 2003/109 del Consejo relativa al estatuto de los nacionales de terceros países residentes de larga duración y Directiva del Consejo 2004/83 por la que se establecen normas mínimas relativas a los requisitos para el reconocimiento y el estatuto de nacionales de terceros países o apátridas como refugiados o personas que necesitan otro tipo de protección internacional y al contenido de la protección concedida. Se ha adoptado una propuesta legislativa 
diendo del instrumento) el acceso al empleo y a la educación y formación o la igualdad de trato9 ${ }^{9}$ La legislación de la UE contra la discriminación apoya este marco jurídico ${ }^{10}$.

Según se anunció en el «Plan de política en materia de migración legal» ${ }^{11}$, la propuesta de Directiva marco general de la Comisión destinada a definir los derechos fundamentales de los trabajadores inmigrantes en la UE y la propuesta de Directiva relativa a las condiciones de entrada y residencia de inmigrantes con alto nivel de formación, son inminentes ${ }^{12}$.

Los beneficiarios de protección internacional requieren medidas adaptadas de integración debido a su situación particular. Este aspecto formará parte del debate puesto en marcha por el Libro verde sobre el futuro sistema europeo común de asilo ${ }^{13}$.

\subsection{Marco de la UE para la integración de nacionales de terceros países}

En 2004, el Consejo Europeo adoptó el Programa de La Haya sobre la consolidación de la libertad, la seguridad y la justicia en la Unión Europea ${ }^{14}$, subrayando la necesidad de una mayor coordinación de las políticas nacionales de integración y de las actividades de la UE sobre la base de principios comunes.

El Consejo adoptó los Principios básicos comunes para una política de integración de los inmigrantes en la $\mathbf{U E}^{15}$ (PBC) y en septiembre de 2005 la Comisión presentó un Programa Común para la Integración que establece un marco para la integración de nacionales de terceros países en la UE ${ }^{16}$. Los elementos clave de este programa son propuestas de medidas concretas para poner en práctica los $\mathrm{PBC}$, tanto en la UE como en los distintos Estados miembros $^{17}$. Además, el Programa Común establece mecanismos de apoyo de la UE para facilitar este proceso mediante el desarrollo de un planteamiento europeo distintivo de la integración a través de la cooperación y del intercambio de buenas prácticas.

con el fin de extender las normas sobre residencia a largo plazo a los beneficiarios de protección internacional; COM (2007) 298.

9 En 2008 se publicará un estudio sobre la conformidad de las medidas nacionales de transposición de las directivas sobre asilo e inmigración.

10 http://ec.europa.eu/employment_social/fundamental_rights/index_es.htm

$11 \operatorname{COM}(2005) 669$

12 La Comisión también presentará propuestas legislativas relativas a los temporeros y los aprendices remunerados en 2008, y a las transferencias dentro de las empresas en 2009.

$13 \operatorname{COM}(2007) 301$

14 Documento 16054/04 del Consejo.

15 Documento 14615/04 del Consejo.

$16 \operatorname{COM}(2005) 389$.

17 El Parlamento Europeo, el Comité Económico y Social Europeo y el Comité de las Regiones acogieroncon satisfacción el desarrollo del marco de integración de la UE en sus respectivos dictámenes. 
Las conclusiones del Consejo sobre el Programa Común apoyaron las principales orientaciones de éste y subrayaron la necesidad de reforzar un planteamiento común de las políticas y medidas de integración ${ }^{18}$.

En estos momentos, en la red de enlaces nacionales participan activamente todos los Estados miembros. La red se ha convertido en un mecanismo efectivo para intercambiar información y definir ámbitos prioritarios y desempeña un papel importante en el refuerzo mutuo de los esfuerzos nacionales y de la UE.

Los Manuales sobre integración para responsables políticos y profesionales $^{19}$, compilados en cooperación con los enlaces nacionales, las autoridades regionales y las organizaciones no gubernamentales, constituyen una guía para el intercambio de información y buenas prácticas.

La primera edición (2004) se refería a la introducción de inmigrantes recientemente llegados y de refugiados reconocidos, a la participación cívica y a los indicadores. La segunda edición (2007) se centra en otras cuestiones clave desarrolladas en los PBC: infraestructura de integración, con un examen de los mecanismos utilizados para ejecutar estrategias acertadas de integración en todos los ámbitos; y alojamiento en medio urbano e integración económica, que presenta las lecciones aprendidas en estos campos. Está prevista una tercera edición para 2009.

Según se pedía en el Programa de La Haya, la Comisión está desarrollando un sitio internet fácilmente accesible, con el fin de apoyar la promoción del intercambio estructural de experiencia e información sobre integración y que estará en funcionamiento en 2008.

Un planteamiento integral que implique a los interesados en todos los niveles es esencial para desarrollar una política de integración efectiva, tal como se recoge en el Programa de La Haya. La puesta en marcha de un proceso de cooperación transnacional a nivel municipal entre poderes públicos, empresas privadas, organizaciones sociales y asociaciones de emigrantes mediante el congreso Ciudades integradoras, celebrado en Rotterdam en octubre de 2006, fue un paso crucial $^{20}$. También está previsto reunir en un Foro Europeo de la Integración a interesados que trabajan en la UE en pro de la integración; en él, grupos de organizaciones que cuentan con miembros en varios Estados miembros intercambiarán experiencias y elaborarán recomendaciones que se incorporarán en un sitio internet dedicado a la integración.

Con el fin de proseguir el debate político iniciado en la primera conferencia ministerial sobre integración que tuvo lugar en Groningen en 2004, en mayo de 2007 se celebró en Potsdam una reunión informal de ministros de la UE responsables de integración y como seguimiento de la misma, en junio de 2007 el Consejo adoptó conclusiones sobre el refuerzo de las políticas de integración en la UE mediante la promoción de la unidad en la diversidad.

18 Documento 14390/05 del Consejo.

19 ec.europa.eu/justice_home/doc_centre/immigration/integration/doc_immigration_integration_en.htm

20 La próxima edición se celebrará en Milán, en el otoño de 2007, organizada por la red Eurocities. 


\subsection{Integración}

Basándose en el marco de integración de la UE apoyado por los PBC y como seguimiento a las sugerencias de medidas concretas a nivel de la UE presentadas en el Programa Común, la Comisión desarrolló un planteamiento más coherente de la integración, que pasa así a ser una parte integrante de la política y la práctica de toda una gama de ámbitos de actuación de la UE.

Para facilitar la incorporación de la inmigración, incluidos los objetivos de integración, en las iniciativas de la Comisión, se creó un Grupo de Comisarios para asuntos de inmigración, con el fin de reunir a todas las áreas políticas afectadas ${ }^{21}$.

El trabajo es una parte clave del proceso de integración y la integración efectiva de los inmigrantes en el mercado laboral constituye una contribución importante para alcanzar los objetivos de Lisboa en términos de empleo y crecimiento. En el contexto de la Estrategia Europea de Empleo, las Directrices integradas instan a los Estados miembros para que consideren la integración de los inmigrantes en los mercados laborales de la $\mathrm{UE}^{22}$. La Comisión hace un seguimiento del impacto de los programas nacionales de reforma mediante informes conjuntos anuales sobre empleo y anima a los Estados miembros a convertir la integración de los inmigrantes en el mercado laboral en una dimensión más explícita de las políticas de empleo ${ }^{23}$. Con el fin de analizar las barreras del mercado laboral e intercambiar buenas prácticas, se creó un Grupo consultivo de alto nivel sobre la integración social de las minorías étnicas y su participación plena en el mercado laboral.

Los inmigrantes representan un grupo importante de posibles empresarios en Europa ya que sus negocios tienen un impacto significativo en el crecimiento económico de la UE. Con el fin de superar las dificultades para la creación de empresas, se creó una Red de empresarios de minorías étnicas. Se publicará un estudio sobre buenas prácticas en este ámbito y está previsto celebrar un congreso en la primavera de $2008^{24}$.

Cada vez se reconoce más la importancia de la dimensión cultural de la integración y el diálogo intercultural, incluido el diálogo entre religiones y en el seno de las mismas, se ha convertido en un instrumento esencial para estimular una integración exitosa y contrarrestar el racismo y el extremismo. El Año Europeo del Diálogo Intercultural (2008) supondrá una importante consolidación de las actividades en este campo.

La promoción de los derechos fundamentales, la no discriminación y la igualdad de oportunidades desempeñan un papel crucial en el contexto de la integración. Un Grupo interservicios sobre racismo y xenofobia coordina las políticas al respecto en el seno de la Comisión y la Agencia de Derechos Fundamentales asesora en este campo $^{25}$. Los esfuerzos para eliminar las ba-

21 ec.europa.eu/commission_barroso/frattini/doc/2006/pr_30_08_06_en.pdf

$22 \operatorname{COM}(2006) 815$.

23 Documento 6706/07 del Consejo.

24 http://ec.europa.eu/enterprise/entrepreneurship/sme_policy.htm

25 eumc.europa.eu/eumc/index.php 
rreras estructurales a las que se enfrentan los inmigrantes se están redoblando en el contexto del Año Europeo de la Igualdad de Oportunidades para Todos (2007), mediante el lanzamiento de un importante debate sobre los beneficios de la diversidad ${ }^{26}$. Como las mujeres constituyen la mayor parte de la población inmigrante en la $\mathrm{UE}^{27}$, sus necesidades específicas se ven cada vez más reflejadas mediante mecanismos de integración del factor de igualdad de sexos en el conjunto de las políticas, tales como el Plan de Igualdad entre Mujeres y Hombres 2006-201028.

La Comisión subrayó la dimensión de integración en las políticas de integración social y protección social, en el marco de los objetivos comunes de la UE, con el fin de que los Estados miembros la traduzcan en sus políticas nacionales y regionales sobre la base de informes nacionales sobre estrategias de protección e integración social. El proceso de supervisión de estas políticas contribuye a los esfuerzos para reforzar medidas de integración que colmen la división existente entre inmigrantes y ciudadanos ${ }^{29}$. En la Comisión, un Grupo interservicios sobre desarrollo urbano coordina la dimensión urbana de las políticas comunitarias ${ }^{30}$ y otras iniciativas urbanas (tales como la Red Europea de Ciudades para la Política e Integración Local, CLIP, coordinada por la Fundación Europea para la Mejora de las Condiciones de Vida y de Trabajo) se han creado para compartir buenas prácticas sobre una amplia gama de asuntos relacionados con la integración. Se presta una atención particular a las necesidades de los grupos vulnerables, con el fin de reducir las desigualdades en el marco del Programa de acción comunitario en el ámbito de la salud pública $(2003-2008)^{31}$.

La educación y la formación facilitan herramientas para mejorar el nivel de los logros y son esenciales para que los inmigrantes participen activamente en la sociedad. La integración se promueve a través de iniciativas educativas tales como el Programa de Educación y Formación 2010 32 . Como los niños y jóvenes de origen inmigrante tienden a obtener resultados escolares inferiores a los de otros alumnos ${ }^{33}$, una próxima comunicación de la Comisión explorará las causas e indicará medidas para hacer frente a estos retos educativos. La integración es un proceso espontáneo, especialmente para los niños. Superar los retos de la integración desde el primer momento supone un planteamiento clave para lograr buenos resultados y la estrategia de la UE sobre los derechos de la infancia ${ }^{34}$ tiene en cuenta el potencial de este grupo. Por otra parte, el diálogo estructurado a nivel de la UE en el contexto de las políticas de ju-

2626 ec.europa.eu/employment_social/eyeq/index.cfm

27 División de Población de la ONU, Trends in Total Migrant Stock: The 2005 Revision.

$28 \mathrm{http}: / /$ ec.europa.eu/employment_social/gender_equality/gender_mainstreaming/general_overview.html

29 Informe conjunto sobre protección social e inclusión social 2007 (register.consilium.europa.eu/pdf/en/07/ st06/st06694.en07.pdf)

$30 \mathrm{La}$ dimensión urbana en las políticas comunitarias (2007-2013); ec.europa. eu/regional_policy/sources/docgener/guides/urban/index_en.htm\#down

31 ec.europa.eu/health/ph_programme/programme_en.htm

32 http://ec.europa.eu/education/policies/educ/eit/index_es.html.

33 PISA 2006, OCDE.

$34 \operatorname{COM}(2006) 367$. 
ventud como seguimiento del Pacto Europeo para la Juventud ${ }^{35}$, contribuye a abordar las necesidades específicas de este grupo destinatario, centrándose en la integración social y profesional de los jóvenes en 2007 y en el diálogo intercultural en 2008.

\subsection{Instrumentos financieros de la UE}

La UE apoya las políticas de integración de los Estados miembros mediante varios instrumentos financieros. Desde 2003, la Comisión ha cofinanciado proyectos transnacionales de integración que promueven la cooperación entre Estados miembros, autoridades regionales y locales y otros interesados mediante las acciones preparatorias INTI ${ }^{36}$.

En el marco del programa Solidaridad y Gestión de los Flujos de Inmigración 2007-2013, el Fondo Europeo de Integración de Nacionales de Terceros Países apoyará los retos de integración a los que se enfrenta Europa. El Fondo pretende crear una nueva forma de solidaridad para promover los esfuerzos de los Estados miembros con el fin de que los inmigrantes puedan cumplir las condiciones de residencia y facilitar su integración. También ayudará a los Estados miembros a compartir sus mejores prácticas, reforzando la cooperación en la $\mathrm{UE}^{37}$.

El Fondo Europeo de Refugiados apoya medidas adaptadas de integración de estas personas, incluidos los beneficiarios de protección subsidiaria cuya estancia en la UE es de larga duración y estable. En el marco del programa Solidaridad y Gestión de los Flujos de Inmigración, el nuevo Fondo Europeo de Refugiados, que se pondrá en marcha en 2008, continuará financiando estos proyectos específicos ${ }^{38}$.

En el contexto del Fondo Social Europeo (FSE), la iniciativa comunitaria EQUAL ofreció un conjunto de buenas prácticas innovadoras para prevenir y luchar contra la discriminación de los inmigrantes en el mercado laboral ${ }^{39}$. La creciente participación de los inmigrantes en el empleo y, en consecuencia, la consolidación de su integración social, es una prioridad específica del nuevo FSE para $2007-2013^{40}$. Por otra parte, el nuevo programa PROGRESS 20072013 también apoyará la aplicación de los principios de lucha contra la discriminación y de igualdad de sexos.

Los instrumentos de política regional también abordan los problemas de inmigración e integración, especialmente en zonas urbanas. En especial, la iniciativa comunitaria URBAN II se focalizó en gran medida en la integración social en zonas urbanas desfavorecidas ${ }^{41}$ y el programa URBACT, de intercambio de experiencias sobre problemas de desarrollo urbano, tiene en

\footnotetext{
$35 \operatorname{COM}(2005) 206$.

3636 ec.europa.eu/justice_home/funding/2004_2007/inti/funding_inti_en.htm

37 ec.europa.eu/justice_home/funding/integration/funding_integration_en.htm

38 http://europa.eu.int/comm/justice_home/funding/refugee/funding_refugee_en.htm

39 http://ec.europa.eu/employment_social/index_es.html

40 ec.europa.eu/employment_social/esf2000/2007-2013_en.html

41 http://ec.europa.eu/regional_policy/urban2/audit_es.htm
} 
cuenta los retos específicos de la diversidad a los que se enfrentan las ciudades europeas. Este planteamiento continuará con el programa URBACT II $2007-2013^{42}$.

\section{EVOLUCIÓN DE LAS POLÍTICAS NACIONALES DE INTE- GRACIÓN ${ }^{43}$}

La integración de nacionales de terceros países ha sido objeto de un debate centrado en los fenómenos de discriminación y diversidad cultural y religiosa. En algunos casos, determinados acontecimientos dramáticos fueron cruciales para influir en la percepción de la inmigración por parte de la opinión pública y muchos Estados miembros definieron nuevas prioridades y revisaron sus políticas. La mayor parte de los conceptos presentes en las políticas de integración de los Estados miembros están codificados por los PBC y, en diversa medida, se reflejan en sus estrategias de integración.

- PBC1. En los Estados miembros se han adoptado diversas medidas para estimular la integración como proceso bidireccional. Sin embargo, aplicar este principio de forma significativa es un reto a largo plazo que requiere otros esfuerzos. Las iniciativas estructurales concebidas para que la población de acogida refuerce su capacidad de ajustarse a la diversidad aún están infrarrepresentadas en las estrategias nacionales.

- PBC2. Valores básicos como la libertad, la democracia, el Estado de Derecho y el respeto de los derechos humanos y las libertades fundamentales se consideran elementos importantes de las nuevas políticas. Varios Estados miembros han introducido medidas para fomentar el conocimiento de los valores básicos mediante programas de orientación cívica.

- PBC3. La integración de inmigrantes en el mercado laboral sigue siendo un reto importante para las políticas nacionales de integración, aunque cada vez se aplican más medidas para consolidar este aspecto: prevención del desempleo a través de la educación y la formación, sistemas más efectivos para reconocer las calificaciones, lucha contra la discriminación en el puesto de trabajo y promoción del empleo de las mujeres inmigrantes.

- PBC4. La mayor parte de los Estados miembros consideran el conocimiento básico de su lengua como un elemento esencial de la integración. Muchos países centran sus estrategias de integración en programas introductorios que a veces incluyen cursos obligatorios de lengua y educación para la ciudadanía destinados a los recién llegados, y un creciente número de Estados miembros está incrementado la flexibilidad de los cursos para centrarlos en necesidades específicas; solamente algunos Estados miembros realizan una evaluación en profundidad de estas actividades.

- PBC5. Las políticas de integración incluyen la educación y la formación como elementos fundamentales del proceso. Los esfuerzos de los Estados

42 urbact.eu/hacia-urbact-2.html

43 Esta sección se ha redactado sobre la base de las respuestas de los Estados miembros a un cuestionario de la Comisión. Véase anexo: Informe resumido sobre las políticas de integración en la UE-27. 
miembros se centran en clases específicas de lengua y tutorías para facilitar la integración en la escuela. Muchas iniciativas promueven el respeto de la diversidad en el ámbito educativo y el apoyo a los profesores. Sin embargo, los niños y jóvenes inmigrantes se enfrentan a retos específicos a los que deberá prestarse más atención.

- PBC6. Aunque un número cada vez mayor de Estados miembros desarrolle la posibilidad de que los prestadores de servicios colaboren con los inmigrantes, otorgan una importancia dispar a este aspecto. En algunos Estados miembros se ponen en marcha iniciativas de igualdad de acceso a las instituciones públicas, incluidas medidas contra la discriminación e informativas. El desarrollo de la cooperación entre instancias estatales y la participación de las empresas en el debate sobre la integración son medidas que solo ahora comienzan a adoptarse.

- PBC7. La importancia de las relaciones en la vida cotidiana y el papel crucial de las actividades locales es subrayada por la mayor parte de los Estados miembros, aunque existen grandes diferencias sobre en qué medida tales actividades se reflejan en las estrategias de integración. Todavía son escasas las medidas para promover iniciativas de interacción entre los inmigrantes y la sociedad de acogida, incluida la creación de foros comunes.

- PBC8. Las legislaciones de los Estados miembros garantizan el respeto de todas las religiones y el principio de no discriminación por razones religiosas. Aunque existe consenso sobre la importancia del diálogo entre religiones y en el seno de las mismas como elemento que coadyuva a iniciativas interculturales más amplias, las medidas para reforzar este aspecto surgen a menudo como respuestas ad hoc a acontecimientos puntuales. En algunos Estados miembros, comienza a promoverse dicho diálogo sobre una base más estructurada.

- PBC9. La participación de los inmigrantes en el proceso democrático se percibe cada vez más como un aspecto significativo de una integración acertada y en un número creciente de casos los representantes de los inmigrantes participan en la elaboración y ejecución de las políticas de integración. En especial, existe un interés cada vez mayor por los procesos activos de ciudadanía y naturalización como elementos para consolidar las oportunidades de participación en la sociedad de acogida. Un número bastante limitado de Estados miembros ofrece a los nacionales de terceros países el derecho de voto en las elecciones locales.

- PBC10. La mayor parte de los Estados miembros han reforzado su capacidad de incluir la integración en todas las políticas pertinentes, aunque también desarrollando medidas específicas. Sin embargo, el intercambio efectivo de información, la coordinación con todos los niveles estatales y los interesados y la atención debida a la igualdad de sexos y a las necesidades específicas de los niños y jóvenes inmigrantes, aún constituyen retos importantes.

- PBC11. Los Estados miembros perciben cada vez más la necesidad de aumentar la capacidad de recopilar, analizar y difundir de forma más sistemática información sobre integración, incluidas estadísticas desglosadas por 
sexo. Unos datos más detallados ayudan a evitar confusiones y a mejorar la percepción de la contribución de los inmigrantes al desarrollo de la sociedad de acogida. También se precisarán otras medidas para supervisar y evaluar las políticas y programas de integración y para identificar indicadores específicos.

\section{CONCLUSIONES}

Las conclusiones del Consejo de junio de 2007 marcaron un nuevo hito en la política de integración de la UE al subrayar la necesidad de considerar que la integración implica a toda la sociedad y de reconocer que el diálogo intercultural es un instrumento importante para estimular la integración.

Para desarrollar el marco de la UE sobre la base de los Principios de Base Comunes y el Programa Común, la Comisión presentará nuevas iniciativas. Los enlaces nacionales desempeñarán un papel esencial en este proceso.

La Comisión explorará diversos conceptos de participación y ciudadanía y su influencia en el proceso de integración. Se promoverán a todos los niveles plataformas de debate en las que participen los interesados y representantes de los inmigrantes.

La Comisión también examinará el valor añadido de módulos europeos comunes para la integración de inmigrantes basados en las buenas prácticas existentes, con el fin de desarrollar directrices sobre diversos aspectos del proceso de integración (cursos introductorios, promoción de la participación de los inmigrantes y de otros ciudadanos en la vida local, etc.).

Existe un consenso sobre la influencia de los medios de comunicación para orientar el debate público, pues pueden contribuir a concienciar, a aclarar malentendidos y a involucrar a unas sociedades cada vez más diversas en un debate productivo. Sobre la base de un reciente estudio ${ }^{44}$, la Comisión examinará las formas de asegurar que las oportunidades que los inmigrantes representan para el desarrollo social, el crecimiento económico y la diversidad cultural son conocidas por el público y reconocidas más ampliamente.

La Comisión también explorará la forma en que los procesos de integración podrían contribuir más activamente a impedir la alienación social y la discriminación contra los inmigrantes, centrándose especialmente en la juventud y en la gestión de la diversidad para evitar casos extremos de rechazo de la sociedad de acogida.

Se precisan criterios relativos a diversos aspectos de la integración para conformar efectivamente las políticas y mejorar el rendimiento gracias a lo aprendido de las experiencias más exitosas. La Comisión examinará la forma de promover más el desarrollo de indicadores e índices comunes para el uso por los Estados miembros con el fin de evaluar los programas de integración y de contar con parámetros de referencia para los análisis comparativos.

44 Inmigración y percepción pública, OAPE 2006. 
Finalmente, la Comisión considerará la forma de rediseñar el Informe anual sobre inmigración e integración para convertirlo en un instrumento actualizado de análisis comparativo de los avances en las políticas de integración gracias a un nuevo concepto que haga de él una herramienta más completa de información y supervisión. Por otra parte, la Comisión continuará siguiendo la aplicación de la legislación pertinente de la CE y su impacto en la integración de los nacionales de terceros países.

\section{ANNEX (FOR INFORMATION)}

\section{Summary Report on Integration Policies in the EU-27}

This Summary Report has been drafted on the basis of a questionnaire completed by the National Contact Points on Integration ${ }^{45}$. The questionnaire refers to third-country nationals who are legally residing in the Member States, both newly-arrived and long-established immigrants and refugees.

The aim of the questionnaire was to gather specific information concerning various dimensions of the integration process in the Member States for the calendar year 2005 and the first half of 2006. The Report is structured along the lines of the Common Basic Principles on integration (CBPs) and in keeping with the Common Agenda for Integration.

The CBPs and the Common Agenda are well known by those directly involved in integration policies at national level. Measures aiming at disseminating them further to a wider group of policy-makers and to civil society are undertaken. In Bulgaria and in the Slovak Republic they are discussed and presented to the broader public. They are increasingly mentioned in official declarations and political statements. Some Member States, such as Austria, the Czech Republic, Denmark and Spain, refer to the CBPs on a regular basis when dealing with immigration and integration issues. In the Czech Republic and Greece, the CBPs enriched the debate leading to the adoption of new legislation. They are also explicitly incorporated in some Member States' programmes. In the Spanish Strategic Plan on Citizenship and Integration, a full text version of the CBPs is reproduced and reference is made to the Common Agenda for Integration. In the consultations held with stakeholders about the content of the Strategic Plan, extensive information was given on integration initiatives taken at the EU level. In Ireland, the CBPs continue to inform the policy making process and all projects submitted for funding from a recently announced Immigrant Integration Fund are required to reflect the CBPs.

\section{1. 'Integration is a dynamic, two-way process of mutual accommodation by all immigrants and residents of Member States'}

In the Czech Republic and Greece, this principle is part of, respectively, the 'Updated Concept of Immigrant Integration' and the new 'Integrated

45 Replies have been received from all Member States except Cyprus and Malta 
Action Plan'. In Slovenia, a 'Unit for cultural rights of minorities and for the development of cultural diversity' was established within the Ministry of Culture to support the better understanding and co-existence of different cultural identities. In Belgium, the French and Flemish Communities set up programmes for intercultural communication and awareness-raising on the rights of foreigners targeting both the host society and immigrants. In Denmark, a fund supports local projects such as the 'Copenhagen Day of Dialogue' including intercultural activities and debates. For the 'Danish Constitution Day', a competition for young people, focused on subjects of democracy and integration and widely covered by the media, is prepared. The Swedish government declared the year 2006 the Swedish Year of Multiculturalism' to promote opportunities for all to participate in cultural life and to create co-operation between various cultural traditions. In Luxembourg, the 'Neighbours' Festival', the 'Festival of migrations, cultures and citizenship' and other multicultural initiatives are organised to promote integration. In Finland, immigrants who obtain Finnish nationality are invited to the 'Theme day of nationality' in the city of Turku. A 'Multicultural personality of the year' and a 'New resident of Turku of the year' are elected. In The Netherlands, primary and secondary schools are encouraged to organise initiatives for the promotion of civic citizenship and integration. Within the new Irish National Action Plan against Racism 'Planning for Diversity', local partnership companies support anti-discrimination and integration initiatives. In the Slovak Republic, the new 'Action Plan to Prevent All Forms of Discrimination, Racism, Xenophobia, Anti-Semitism and Other Forms of Intolerance' was adopted. Measures involving the media to promote understanding of immigration are undertaken actively in Belgium, Ireland, Latvia, Lithuania, Luxembourg, Portugal, the Slovak Republic, Spain, Sweden, The Netherlands and the United Kingdom. In Latvia, the 'International Tolerance Day' was organised in collaboration with the media. In Lithuania, a new version of the 'Code of Ethics of Journalists and Publishers' was approved to shape understanding of diversity. In Portugal, many initiatives are carried out to manage cultural diversity including television and radio programmes, such as the 'Week of Cultural Diversity'. In the United Kingdom, the 'Improving Opportunity, Strengthening Society' strategy and the 'Community Cohesion Toolkit' focusing on the role of the media are among the measures launched to foster a sense of common belonging.

\section{2. 'Integration implies respect for the basic values of the European Union'}

A number of Member States, including Belgium, Denmark, Finland, France, Germany, Luxembourg and The Netherlands refer to the basic values of the European Union in introductory programmes for newly-arrived third-country nationals. Some countries increasingly promote EU basic values through broader initiatives. In Belgium, a 'Committee of Seven Wise Men' elaborated on basic values and presented its recommendations to the Flemish government to harmonise civic integration courses. The French Community supports educational programmes targeting school teachers and students. Interdisciplinary citizenship courses will be included in the curriculum of all 
mandatory educational programmes. In France, the concept of integration includes a strong political and civic dimension reflecting common republican values which are discussed by the 'High Council of Integration.' In Lithuania, civic orientation and integration courses on the host society's culture and history are organised for people granted asylum. In Luxembourg, compulsory civic education courses are provided to those applying for nationality. In Bulgaria, a project 'Civic education - road to Europe' is organised targeting young people. Sweden set up initiatives to raise awareness on basic values including a new 'National Action for Human Rights', as well as a specific programme to combat violence and oppression in the name of honour. In The Netherlands, a declaration of 'Solidarity with The Netherlands', covering respect for common values, is pronounced during naturalisation ceremonies.

\section{3. 'Employment is a key part of the integration process and is central to the participation of immigrants, to the contributions immigrants make to the host society, and to making such contributions visible'}

The contribution of immigrants to the economic growth and development of the host society is increasingly recognised, as underlined by Greece, Italy and Spain. To facilitate the labour market integration of immigrants the Czech Republic has planned a simplification of bureaucracy and the possibility to reside legally while looking for a job. In Spain, a new system 'Catalogue of Labour Shortages in Specific Occupations' has been set up to identify shortages and to allow for a swift processing of residence and working permits. Portugal established 'Offices of Employment and Entrepreneurial Support for Immigrants' within the 'National Immigrant Support Centres', and it launched an advertising campaign 'Immigrant Portugal, Tolerant Portugal'. In Poland, a number of labour market integration measures are carried out by the Intercultural Centre for Vocational Adaptation and the Work Club of the Polish Humanitarian Organisation. In the Slovak Republic, the process of assessment of qualifications and skills and the access to vocational training has been simplified. A specific web-site has been created to advertise vacancies and provide information to employers. The Danish government concluded a political agreement 'A new change for everyone' on access to jobs and education, including new financial incentives to municipalities and obligations for local authorities to provide job offers. In Ireland, publications such as the 'Know before you go' booklet including information on finding employment for newly-arrived immigrants and the 'Employment Rights Information Booklet' are available in multiple languages. The 'Employment for Parents of Irish Born Children Programme' promotes the employment of third-country national parents of an Irish born child and the study 'An Exploration of Local Strategies for the Integration of Migrant Workers and their Families' was carried out. In France, a group of big enterprises drafted a 'Diversity Charter', which is now being signed by many other employers, to commit themselves to create an intercultural environment among their staff. The Finnish Ministry of Trade and Industry established a working group to reinforce immigrant entrepreneurship through networking, interaction, education and training, development of advisory services and information. In The Netherlands, an action plan was adopted to further develop immigrant 
entrepreneurship and a monitoring system against discrimination at the work place is being set up. A 'Diversity Unit' was established by the Belgian federal government to fight against discrimination at work and to promote equality. The Flemish Community organises individual labour market insertion programmes for newlyarrived third-country nationals as part of introductory programmes. In Greece, interventions in favour of unemployed immigrant women are a priority. In Sweden, the employment office for immigrants created a special team to provide support before and during the initial period in a new job. Austria promotes employment of immigrants in the public sector. In the United Kingdom, within certain industry sectors, language teachers and integration experts are available to develop schemes for labour market integration of immigrants.

\section{4. 'Basic knowledge of the host society's language, history, and institutio- $n s$ is indispensable to integration; enabling immigrants to acquire this basic knowledge is essential to successful integration'}

In most of the Member States, including Austria, the Czech Republic, Denmark, Germany, Greece, Italy, Latvia, Luxembourg, Portugal and Sweden, information material and welcome packages in various languages are available for newly-arrived third-country nationals. Introduction programmes are established in most Member States and they are compulsory in some countries, i.e. Austria, Belgium, Denmark, France, Germany, Greece and The Netherlands. In Austria, immigrants have to sign an Integration Agreement and to follow German language training in order to receive a residence permit. The City of Vienna provides special cheap courses for young and long-term immigrants. In Denmark, an examination on Danish society, history and culture has to be taken to obtain Danish citizenship and a basic civic test will also be introduced for some categories of immigrants. In the United Kingdom, the 'Life in the United Kingdom: A Journey to Citizenship' syllabus to prepare for the citizenship test was published. In Portugal, the 'Portugal Welcomes You' programme provides language and introductory citizenship courses for newly-arrived thirdcountry nationals. In France, knowledge of the French language, values and institutions is a pre-condition to acquire a long-term residence permit. Language courses, including an examination and a certificate, are compulsory depending on the level of knowledge and they are free of charge for newly-arrived third-country nationals. They are organised in a flexible way according to different needs and child care is available during classes. In Germany, language classes are compulsory depending on the level of knowledge and integration courses provide orientation and basic knowledge of German institutions. In Lithuania, Slovenia, Romania and the United Kingdom a personal integration plan is drafted for every refugee. Italy and Spain foresee introduction courses with a focus on vocational training in their territories, as well as in the immigrants' countries of origin. The Netherlands organises compulsory pre-departure examinations on language and civic orientation for immigrants, with the exclusion of refugees and asylum seekers, to be taken in the country of origin. In Ireland, citizens' information centres are located in every town and cultural orientation programmes and information leaflets are also available. Stakeholders were involved in the organisation of 
language courses to provide an effective service. In Luxembourg, a pilot project for language classes in French and Luxembourgish 'Cours Inlux' has proved to be very successful and will be renewed. In Poland, a web-site was set up after consultation with refugees, in order to provide them with the most useful information and a newspaper addressing immigrants is drafted with their contribution.

\section{5. 'Efforts in education are critical to preparing immigrants, and particu- larly their descendants, to be more successful and more active partici- pants in society'}

In Austria, intercultural teaching and learning are principles of federal law, implemented by providing training and support to teachers and promoting antidiscrimination activities in all schools. In Finland, municipalities are granted an increased State subsidy to support young immigrants speaking other languages and teaching in their mother tongue is available. The general school programme includes teaching on foreign cultures. In Spain, half of the 'State Fund for Reception, Integration and Education' is used by schools to develop reception programmes for newly-arrived young immigrants and their families, to provide teaching support during an initial period and to hire intercultural mediators. Teaching exchange programmes with third countries and a movie 'Settlers' on interculturality at school are examples of initiatives undertaken. In Belgium, the French Community develops courses in the language and culture of origin of immigrants and courses promoting openness towards other cultures. In all parts of the country, reception programmes, bridging classes and language courses are organised to facilitate the introduction of newly-arrived young thirdcountry nationals. In Bulgaria, the national programme for the development of education and training foresees specific measures targeted at children speaking another language. Data and research on performance at school are analysed. In Estonia, relevant resources are allocated to train teachers of Estonian as a second language and for bilingual education. In Romania, free courses of Romanian are available for adults and training for teachers is organised to address better the need of students. Finland and Hungary provide classes in various languages as preparatory courses for the integration of immigrant children into the general education system. Greece organises reception and tutorial classes to ease the integration into schools and other measures are set up to ease enrolment and to support families. In Luxembourg, a reception unit for young immigrants who arrive in the course of the school year was created and intercultural mediators and special staff are available to help with language difficulties. In Portugal, the 'Choices Program' aims at preventing low level of achievements and early school-leaving and the 'Between Cultures Secretariat' promotes intercultural education within the wider educational system by training of teachers. Classes of Portuguese as a second language are available in schools. In the United Kingdom, within the framework of the 'Aiming High' strategy, funding and guidance materials are provided to local authorities and school boards in order to address the needs of immigrant children and youth. In The Netherlands, schools and local authorities organise meetings at least once a year to avoid segregation and to promote integration. In Poland, training for teachers is available and a conference was organised on 
measures tackling language difficulties of immigrant children. A kindergarten project 'Children of the World' addresses integration challenges at an early stage with the interaction of children of different origins through arts and games. In Ireland, the 'Department of Education and Science' established a steering committee to co-ordinate responses to the educational needs of newlyarrived young immigrants and to put in place a system of language support for non-English speakers. New 'Guidelines on Intercultural Education in Primary School' are published to support teachers and school management in developing a more inclusive classroom environment. In Sweden, a specific curriculum for learning Swedish as a second language exists and the 'Higher Education Act' requires higher education institutions to promote actively recruitment of immigrants. In France, language tuition and introductory courses are available at school. Recent measures focus on the participation in preparatory courses for university studies and on the smooth transition into the labour market.

\section{6. 'Access for immigrants to institutions, as well as to public and private goods and services, on a basis equal to national citizens and in a non- discriminatory way is a critical foundation for better integration'}

In Italy, access to social services for immigrants is eased by mediatory services. Legal advice, information and orientation desks are available at the local level. In Lithuania, access to public offices is facilitated by interpretation services and information in other languages. In Austria, special multi-language information desks are available for various services. The User Panel of the Danish Immigration Service is set up to bring together the Danish Immigration Service and immigrant's representatives. In Poland, training is organised for people working with refugees and a centre provides information on legislation and available services within public institutions. In Sweden, all government agencies have to adopt action plans to take into account cultural diversity in recruitment procedures and a system with depersonalised applications for jobs will be tested. In The Netherlands, an anti-discrimination project within the structures of the government is launched. In Finland, the Ministry of Labour produced a 'Handbook on equality data' containing good anti-discrimination practices. In the Czech Republic, multicultural education and improvement of language skills of public administration staff is an increasing priority. Bulgaria implements a training project to teach social assistants to work in multiethnic environments. In Romania, public services may employ interpreters and cultural mediators and train their staff to ease the access of immigrants to services. In the Slovak Republic, training for the staff of labour, social and family affairs offices is available, as well as consulting and mediatory services. In Hungary, desk officers of family support centres and social and labour offices participate in training on integration issues. In Latvia, a 'Centre of trust' is being built for third-country nationals as a contact point with the institutions. In Ireland, the newly established 'Irish Naturalisation and Immigration Service' acts as a one-stop-shop providing a single access point to services. Interpretation support, anti-racism and intercultural training is available for service providers. Government Departments and State Agencies provide information, in multiple languages, on the rights and entitlements of access to a wide range of public services. In Portugal, national and local immigrant support centres are one- 
stop-shops set up for the delivery of services with the involvement of sociocultural mediators. An 'SOS Service for Immigrants' and a simultaneous translation service provide help and information in various languages with the assistance of socio-cultural mediators. Within the pilot project 'Strategic Upgrade of National Refugee Integration Services' (SUNRISE), in the United Kingdom a personal caseworker provides information and advice to facilitate access to services.

6.7. 'Frequent interaction between immigrants and Member State citizens is a fundamental mechanism for integration. Shared forums, intercultural dialogue, education about immigrants and immigrant cultures, and stimulating living conditions in urban environments enhance the interactions between immigrants and Member State citizens'

Some Member States, such as Greece and Italy, stress in particular the role of cultural mediators, as well as of volunteering and third sector organisations facilitating the interaction between immigrants and the host society. In Austria, a special department for integration and diversity matters forms a point of co-operation between immigrants' organisations, nongovernmental organisations and the City of Vienna. In Ireland, local community groups are funded to provide day-to-day support and to promote participation of newly-arrived thirdcountry nationals in local community life. In Bulgaria, the 'Sports Vacation Programme' promotes tolerant inter-ethnic relations. In Estonia, an employment exchange programme between different regions of the country is developed to promote interaction. In Denmark, the participation of immigrants in volunteering organisations is promoted. In Lithuania, a website for various minorities' organisations is created to reach a broader public. In Luxembourg, a pilot project to organise entertainment workshops for the interaction of national citizens with immigrants has been successful and will be repeated in many towns. In The Netherlands, many projects in the context of the 'Broad Initiative for Social Cohesion' have been launched including the 'Not beside but with each other' campaign to avoid segregation. A major project to collect examples of co-operation among young people of different cultures is broadly covered by the media. In the United Kingdom, measures to engage together more closely immigrants and the host population include an action plan on intercultural dialogue, a government 'Respect Task Force' and the cohesion guidance 'Leading Cohesive Communities - a guide for leaders and chief executives'.

6.8. 'The practice of diverse cultures and religions is guaranteed under the Charter of Fundamental Rights and must be safeguarded, unless practices conflict with other inviolable European rights or with national law'

Denmark set up various initiatives fostering intercultural dialogue and stressing religious diversity, including dialogue meetings between the Danish Prime Minister and the Minister for Integration and various ethnic minority organisations. Germany organised a federal level conference to launch a longterm dialogue process with representatives of Muslim communities. In Finland, 
a working group on intercultural and inter-religious dialogue was established within the 'Advisory Board for Ethnic Relations'. It acts as a permanent forum for discussion and exchange of information between religious communities and national authorities to increase mutual understanding. In Italy, a 'Council for Italian Islam' was set up to support the central government in gaining an insight on problems faced by Muslim communities and to establish a permanent dialogue. In Latvia, an anti-discrimination project 'Information campaign against Islamophobia' is being implemented and an on-line encyclopaedia on religious diversity and postcards on inter-religious dialogue have been prepared. In Luxembourg, a public conference is organised every year by an inter-religious group representing all major faiths. In Sweden, the Minister responsible for religious affairs holds regular meetings with representatives of different religious communities aimed at reinforcing mutual understanding and trust. In The Netherlands, training for spiritual leaders is organised by Muslims' organisations and the Ministry of Education, Culture and Science and the Ministry for Immigration and Integration.

6.9. 'The participation of immigrants in the democratic process and in the formulation of integration policies and measures, especially at the local level, supports their integration'

In Belgium, the Czech Republic, Finland, Hungary, Ireland, Lithuania, Luxembourg, Portugal, the Slovak Republic, Slovenia, Spain, Sweden and soon in Greece, third-country nationals are, in principle, entitled to vote in local elections. However, legislative frameworks regulating political participation vary widely. In most of these countries, such voting rights are linked to the length of regular stay or are only recognised to specific categories of thirdcountry nationals. In other Member States, they are only granted to citizens of third countries with which specific bilateral arrangements exist. In Luxembourg, awareness-raising campaigns will be organised to inform immigrants about their voting rights at the local level and a proposal has been presented to extend the competences of municipal advisory councils for foreigners ('Commissions communales consultatives') to favour their participation in public life. In Belgium, the Walloon and Brussels Capital governments encouraged thirdcountry nationals to register to vote in local elections. Cities and communities with a high concentration of minorities are obliged by the Flemish government to facilitate their participation in local policies through special consultative councils and activities of the 'Forum for Ethnic Minorities'. In a growing number of countries, specific support for immigrant women organisations is provided. The Czech Republic, Denmark, Estonia, Greece, Italy, Portugal and Spain involve immigrants' representatives, as well as other civil society actors and different stakeholders, in the elaboration and/or implementation of integration policies. In Portugal, the Consultation Council for Immigration Affairs (COCAI), consisting of five immigrants communities, plays an important role in drawing immigration policies. A 'Council for Ethnic Minorities' has been established in Denmark to advise the Ministry of Integration and integration councils are active at the local level. In Spain, the 'Forum for the Integration of Immigrants', a threetiered consultative body created by the government, involving immigrants' 
associations, social partners, non-governmental organisations and public administration, was renewed with enlarged composition and competences. Its consultation has become mandatory for any legal or practical initiative in the field of integration at national level. In France, the 'National Council for the Integration of Immigrant Population', including representatives of immigrants' associations, has been reestablished and is regularly consulted by the Ministry in charge. In Ireland, funding is provided to immigrants' organisations to promote their participation in the democratic process. Irish partnership companies also facilitate the establishment of local level forums which enhance dialogue and interaction between relevant service providers, representatives of the community and voluntary sector and representatives of immigrants' communities. In Italy, a 'Council dealing with third-country nationals and their families' will be established at national level and 'Immigration Territorial Councils' are set up at local level. In Sweden, government funding has been made available to create a network of elected representatives from municipalities and County Councils to promote an intercultural environment. In the United Kingdom, a 'Commission on Integration and Cohesion' has been set up, involving various stakeholders, to elaborate practical steps in order to make local communities more cohesive and integrated.

6.10. 'Mainstreaming integration policies and measures in all relevant policy portfolios and levels of government and public services is an important consideration in publicpolicy formation and implementation'

In the Czech Republic, all relevant Ministries apply integration mainstreaming in the development of departmental policies and legislation. In the French Community of Belgium, all Ministers commit themselves to undertake concrete actions to promote cultural pluralism and mutual comprehension. In Finland, an integration advisory board was established to coordinate activities of various Ministries. 'The Integration Act' was amended so that measures focusing on public general services take into account the needs of immigrants. In Greece, an inter-ministerial committee, supported by a special committee involving experts, was established by the Minister of Interior, Public Administration and Decentralisation to improve the level of co-operation and co-ordination on integration. In Romania, inter-institutional cooperation is developed by co-ordination meetings organised with representatives of various governmental institutions and non-governmental organisations. Ireland established the 'Irish Naturalisation and Immigration Service' to bring together the various strands of government activity. Within these revised structures, a new 'Integration Unit' is being established to promote and co-ordinate all social and organisational measures. France strengthens efforts in terms of public services and social measures in the areas where immigrants are more present. 
6.11. 'Developing clear goals, indicators and evaluation mechanisms are necessary to adjust policy, evaluate progress on integration and to make the exchange of information more effective'

In Denmark, a study on integration indicators is under way and benchmarking of integration efforts at municipal level is published every year by the Minister of Refugee, Immigration and Integration Affairs to identify best practices. The Swedish 'Integration Board' monitors the situation and progress of integration policies, analysing various aspects of Swedish society and producing yearly reports to assist decision-making in this field. In Portugal, the 'Immigration Observatory' carries out research to support the development of integration policy. In Romania, comprehensive research on the situation of persons granted protection, including the analysis of many integration aspects, has been carried out. Its policy recommendations will be used for drafting future measures. Germany and Estonia selected external contractors to evaluate their integration programmes in order to inform future policymaking and enable adjustments for more effective policy outcomes. In Estonia, a public opinion survey was also carried out. In Ireland, all State funding initiatives on integration have clear performance indicators. Procedures are in place to monitor the implementation of funded projects and to inform developing integration policy. In the Czech Republic, within the 'Commission for the Integration of foreigners' one of the working groups deals also with the setting up of integration indicators. 